\title{
Emodialisi domiciliare: obiettivo efficace in un determinato contesto, difficilmente realizzabile nel contesto attuale
}

\author{
Giuliano Giachino
}

\author{
Servizio di Nefrologia e Dialisi, Ospedale "Nuovo", Rivoli, Torino
}

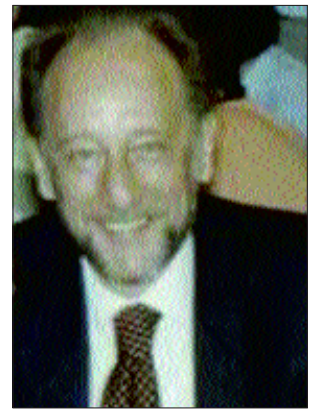

I In una fredda serata dell'ormai lontano autunno del 1972 veniva effettuata a Torino la prima (o una delle prime, poiché vi fu una quasi contemporaneità con la medesima iniziativa parallela messa in atto da colleghi nefrologi di altra città) emodialisi domiciliare d'Italia.

Tre giovani assistenti del professor Antonio Vercellone, tra cui il sottoscritto, erano presenti all'inizio dell'applicazione; e una volta avviatala, trattandosi di una dialisi lunga e notturna, tirarono a sorte tra di loro chi dovesse fermarsi per la notte presso il domicilio del paziente: il fiammifero più corto toccò a me, e fui quindi io a rimanere.

Anni dopo, tra il 1975 e il 1979, il sottoscritto ricoprì il compito di occuparsi del programma di emodialisi domiciliare della Divisione di Nefrologia e Dialisi dell'Ospedale San Giovanni Battista di Torino, diretta dal professor Vercellone, programma che giunse a conta- re oltre 50 pazienti; successivamente ancora, tra il 1980 e il 1985 , trasferitomi presso la Divisione di Nefrologia e Dialisi dell'allora sede Nuova Astanteria Martini diretta dal Prof. G. Piccoli (oggi Ospedale G. Bosco) collaborai a lungo con il collega responsabile del locale programma di emodialisi domiciliare, che arrivò a contare oltre 100 pazienti trattati presso il proprio domicilio, sparsi su tutto il territorio della Regione Piemonte e anche su quello nazionale.

Questa specie di "amarcord" altro scopo non ha, se non quello di sgombrare preventivamente il campo da un certo numero di dubbi: il sottoscritto è stato a suo tempo uno dei più accaniti e convinti assertori dell'emodialisi domiciliare, e ha personalmente selezionato, convinto, istruito, instradato, inviato a domicilio e seguito clinicamente dopo l'inizio della loro emodialisi domiciliare decine e decine di pazienti.

Oggi, a moltissimi anni di distanza, le mie idee e i miei convincimenti in proposito si sono in gran parte modificati: non certamente per quel che riguarda gli aspetti positivi dell'emodialisi domiciliare, che sempre gli stessi sono e conservano di per sé la loro validità, ma bensì circa il contesto in cui una rinata emodialisi domiciliare verrebbe oggi a porsi, contesto profondamente mutato da allora e tale, a mio parere, da rendere un ipotetico tentativo di resuscitarla un'operazione difficilmente realizzabile perché non sussistono più le condizioni necessarie a un suo successo. Sappiamo tutti perfettamente quali siano le ragioni che hanno portato al progressivo decadere dell'emodialisi domiciliare nell'ultimo ventennio. In ordine sparso: il progressivo invecchiamento della popolazione introdotta in dialisi; la progressiva riduzione percentuale dei casi in condizioni cliniche sufficientemente buone a consentirla; la nascita e l'affermazione degli Ambulatori extraospedalieri tipo "self" e successivamente ad assistenza limitata; il maggiore drop-out verso il trapianto rispetto ad altre modalità dialitiche; il prendere piede di tipi di dialisi domiciliare alternative, come la dialisi peritoneale nelle sue diverse modalità, e altre ancora.

In questo contesto, quali possono, oggi, essere le ragioni valide per propugnare una rinascita dell'emodialisi domiciliare e le concrete possibilità di successo di un programma di questo tipo? 
- Una particolare abbondanza di pazienti clinicamente e psicologicamente adatti a praticare l'emodialisi domiciliare?

Non direi proprio: i pazienti clinicamente adatti a una emodialisi domiciliare sono oggi, a mio parere, una vera rarità, e questa rarità è confermata e ribadita dal fatto che sta oggi diventando problematico perfino reperire pazienti in condizioni cliniche sufficientemente buone per poter essere decentrati presso ambulatori extraospedalieri ad assistenza limitata, sedi, queste ultime, che cominciano a vedere attivati, al fine di un loro sfruttamento ottimale, dei turni dialitici assistiti dal personale medico.

Una così marcata scarsità di pazienti adatti rende a mio parere dispersivo di potenzialità altrimenti meglio sfruttabili il notevole sforzo organizzativo necessario ad attivare un programma di emodialisi domiciliare, coinvolgente medici, infermieri, tecnici, amministrativi e tutti i relativi uffici aziendali.

Tutto ciò non vieta affatto, anzi, che un paziente particolarmente adatto non possa e non debba essere doverosamente appoggiato, come mi è accaduto di fare in tempi recenti, a un Centro dialisi che disponga di un programma di emodialisi domiciliare ancora attivo, e che non debba quindi instradare e superare ex novo tutti i problemi soprasegnalati a fronte di un numero assai limitato di casi da trattare.

- Una grave carenza di posti per la dialisi extracorporea presso la sede ospedaliera del Centro, elo presso gli eventuali ambulatori extraospedalieri ad assistenza limitata?

È questo certamente un problema importante e molto sentito un po' dovunque: ma per farvi fronte disponiamo della dialisi peritoneale, anch'essa domiciliare e mediamente ancor meno costosa dell'emodialisi domiciliare.

- Una migliore qualità di vita per il paziente?

Senza dubbio, ma una qualità di vita superiore in grado simile può essere offerta dalla dialisi peritoneale domiciliare, e di poco inferiore dalla dialisi extracorporea ad assistenza limitata presso ambulatori extraospedalieri decentrati e più propinqui del Centro ospedaliero al domicilio del paziente. $\mathrm{E}$ questo anche senza voler tenere conto di una sia pur limitata percentuale di casi, di fronte ai quali qualsiasi nefrologo che si sia dedicato per anni all'emodialisi domiciliare, come il sottoscritto, si è trovato a meditare, assistendo all'evolversi di amari drammi familiari innescati proprio dall'irruzione del trattamento all'interno della famiglia e del domicilio.

- Una migliore riabilitazione sociolavorativa?

Anche qui, certamente. Ma ciò può essere ottenuto facilmente anche attivando, presso gli ambulatori extraospedalieri ad assistenza limitata, degli appositi turni serali, mirati a pazienti che conservino una normale attività lavorativa: ciò è routinariamente messo in atto presso alcuni degli ambulatori ad assistenza limitata dipendenti dal Servizio di Nefrologia e Dialisi da me diretto, ed è mia intenzione estendere ulteriormente in futuro il ricorso a questa modalità di trattamento. - Un minor costo del trattamento? Anche qui, senza alcun dubbio, sì. Per contro, anche senza voler insistere sul fatto che la dialisi peritoneale domiciliare costa ancora meno, credo che non dovremmo dimenticare il fatto che risparmi consistenti nel campo della dialisi sono perseguibili e raggiungibili anche con altri mezzi, che non scarichino sul paziente parte del loro "costo" in termini di coinvolgimento: a esempio, com'è oggi personale esperienza positiva del sottoscritto presso la propria sede lavorativa, il riuscire a erodere gli ampi margini a tutt'oggi ancora esistenti circa il costo dei materiali di consumo; l'adozione, in alternativa agli acquisti tradizionali, di Gare di Service globali comprensive di materiali di consumo, attrezzature, impianti di depurazione delle acque, arredi e assistenza tecnica; l'adozione di sistemi innovativi di pagamento "a posteriori" e "ad applicazione effettuata", in grado di minimizzare gli sprechi di materiale e giacenze inutilizzate di magazzino; il contrarre al minimo indispensabile la percentuale di applicazioni a più elevato costo, non sempre giustificate dalle effettive necessità del paziente, e così via.

Mi rendo purtuttavia conto di come il minor costo dell'emodialisi domiciliare resti, anche al di là di queste mie argomentazioni, un elemento molto forte in suo favore, in specie nel contesto sociale attuale di ripetuti e accaniti tagli dei finanziamenti destinati alla Sanità, di tendenza a una sua progressiva privatizzazione, di capitalismo senza più argini, di globalizzazione selvaggia, di progressiva deriva verso modelli socioeconomici d'Oltreoceano.

Io, ormai in compagnia di pochi intimi in questa mia posizione di "retroguardia", continuo tuttavia a illudermi e a pensare che la tutela della salute dovrebbe venir garantita ai cittadini dallo Stato, certamente non trascurando l'aspetto economico, ma non ponendolo neppure ai più elevati livelli di priorità.

In definitiva: sì, senza dubbio, all'obiettivo di indirizzare nuovamente all'emodialisi domiciliare quell'esiguo numero di pazienti con caratteristiche con essa compatibili, e che oggi forse non se la vedono più proporre; ma accorpandoli presso un numero limitato di Centri dialisi già attrezzati per la gestione di una tale attività, e senza perdere di vista tutte le altre possibilità in grado di contribuire, assieme a essa, a una contrazione dei costi del trattamento.

dialisi.riv@asl5.piemonte.it 